\title{
Optimización de la eficiencia de la celda fotovoltaica
}

\section{Optimization of the efficiency of the photovoltaic cell}

\author{
SANDOVAL-VILLEGAS, Juan Carlos $\dagger^{*}$, TOLOSA-GARMA, Irving Manuel y QUINTAL- \\ VÁZQUEZ, José Francisco
}

Universidad Tecnológica de Cancún, División de Ingeniería y Tecnología.

ID $1^{\text {er }}$ Autor: Juan Carlos, Sandoval-Villegas / ORC ID: 0000-0002-4767-4059, CVU CONACYT ID: 343213

ID $1^{\mathrm{er}}$ Coautor: Irving Manuel, Tolosa-Garma / ORC ID: 0000-0003-3765-0050, CVU CONACYT ID: 484309

ID $2^{\text {do }}$ Coautor: José Francisco, Quintal-Vázquez / ORC ID: 0000-0002-5071-6459, CVU CONACYT ID: 306762

DOI: $10.35429 /$ JEE.2019.9.3.21.28

Recibido Marzo 20, 2019; Aceptado Junio 30, 2019

\section{Resumen}

La eficiencia de producción eléctrica de una celda solar se ve afectada por su temperatura de operación: al incrementar esta la eficiencia disminuye. El propósito del presente trabajo es demostrar tal efecto y cómo el uso de disipadores de calor ayudan a mejorar la eficiencia en las celdas fotovoltaicas a temperaturas superiores a las de diseño (NOCT $20^{\circ} \mathrm{C}$ ). El experimento consistió en medir la temperatura ambiente, la temperatura de la superficie de la celda solar, velocidad del viento, voltaje, corriente y potencia de la celda solar, al aplicarle una carga electrónica controlada. Estas variables se registrarán para una celda solar, adjuntándole un disipador de calor en la cara posterior y para una celda solar sin disipador. Las mediciones permitieron obtener la curva corrientevoltaje de ambas celdas, que revela que la celda con disipador de calor es más eficiente en la producción de energía que la celda sin disipador de calor en un rango entre 3.4\%-5.4\%. El estudio fue desarrollado en la ciudad de Cancún, Quintana Roo, con una temperatura media de la superficie del panel de $40.42^{\circ} \mathrm{C}$, irradiación solar promedio de $675 \mathrm{~W} / \mathrm{m}^{2}$ y una velocidad promedio del viento de $1.88 \mathrm{~m} / \mathrm{s}$.

Celda Fotovoltaica, Eficiencia, Potencia

\begin{abstract}
The electrical production efficiency of a solar cell is affected by its operating temperature: when it increases, efficiency decreases. The purpose of this paper is to demonstrate this effect and how the use of heat sinks help to improve the efficiency in photovoltaic cells at temperatures higher than those of design (NOCT $20^{\circ}$ C). The experiment consisted in measuring environmental temperature, cell's surface temperature, wind speed, voltage, current and power production of the solar cell while an electronically controlled load was connected(or demanding .01A). These measures were taken from two solar cells, one of them with a heatsink and the other (or another) without it. The measurements allowed us to obtain the current-voltage curve of both cells, which reveals that the cell with heat sink is more efficient in the production of energy than the cell without heat sink in a range between $3.4 \%-5.4 \%$. The study was developed in the city of Cancún, Quintana Roo, with an average surface temperature of the panel of $40.42{ }^{\circ} \mathrm{C}$, average solar irradiation of $675 \mathrm{~W} / \mathrm{m}^{2}$ and an average wind speed of $1.88 \mathrm{~m} / \mathrm{s}$.
\end{abstract}

Photovoltaic cell, Power, Efficiency

Citación: SANDOVAL-VILLEGAS, Juan Carlos, TOLOSA-GARMA, Irving Manuel y QUINTAL-VÁZQUEZ, José Francisco. Optimización de la eficiencia de la celda fotovoltaica. Revista de Ingeniería Eléctrica. 2019. 3-9: 21-28

\footnotetext{
$\dagger$ Investigador contribuyendo como primer Autor.

*Correspondencia al Autor correo electrónico: (jsandoval@utcancun.edu.mx)
} 


\section{Introducción}

La producción de energía eléctrica a través de celdas solares, requiere del fenómeno fotovoltaico, que genera un voltaje de circuito abierto y no hay un flujo de corriente eléctrica en el circuito externo, es el voltaje máximo que la celda solar puede producir, que se expresa con la relación: $\operatorname{Voc}(T)=V o c+\frac{d V o c}{d T}\left(T-T_{S T C}\right)$

Donde se observa que su voltaje en circuito abierto está a razón del cambio de temperatura. Por otra parte, el rango común de eficiencia de las celdas solares comerciales está entre 12 y $19 \%$ y los factores que permiten su variación son: la temperatura, la exposición a la luz solar, las propiedades de los rayos solares, suciedad, polvo, entre otros. (S. Mekhilef, 2012/6.). La forma de mejorar la eficiencia de las celdas solares es disminuyendo su temperatura de operación, para que se mantengan operando en el rango de especificaciones técnicas del fabricante.

Para la ciudad de Cancún, la irradiancia para un día despejado es de $5.8 \mathrm{kWh} / \mathrm{m}^{2}$, que es la insolación diaria promedio y se expresa en horas solares pico (HSP), (Lesage, 2017) temperatura ambiente máxima de $33.6^{\circ} \mathrm{C}$ (Organización meteorológica mundial, 2019), es conveniente mantener los paneles solares dentro de sus especificaciones de operación. El propósito es mejorar la eficiencia de la celda solar, instalando en su cara posterior un disipador de calor que pueda descender la temperatura de operación. Es decir, al disminuir la temperatura de operación de la celda solar, se incremente su eficiencia.

Se hace una revisión de la eficiencia de las celdas solares, se describe las relaciones y variables del voltaje en circuito abierto. Se utilizó una metodología teórico-práctica; describiendo el proceso de ubicación y método de medición de los parámetros de la celda solar, se ilustra el comportamiento de la potencia eléctrica, el viento y la temperatura de la celda solar y cómo se relacionan éstas. También se utilizaron estadísticos de prueba para conocer si la producción promedio de potencia de la celda solar, son iguales usando el disipador de calor y sin disipador de calor.

\section{Estado del arte}

De acuerdo con (Arno Smets, 2016) si la temperatura incrementa manteniendo la irradiación solar constante, el voltaje y corriente disminuyen, efecto que se puede observar en la Figura 1. Este efecto recibe el nombre de coeficiente de temperatura de una celda solar, medida especificada por el fabricante que hace referencia a la tasa de cambio del voltaje con respecto a la temperatura.

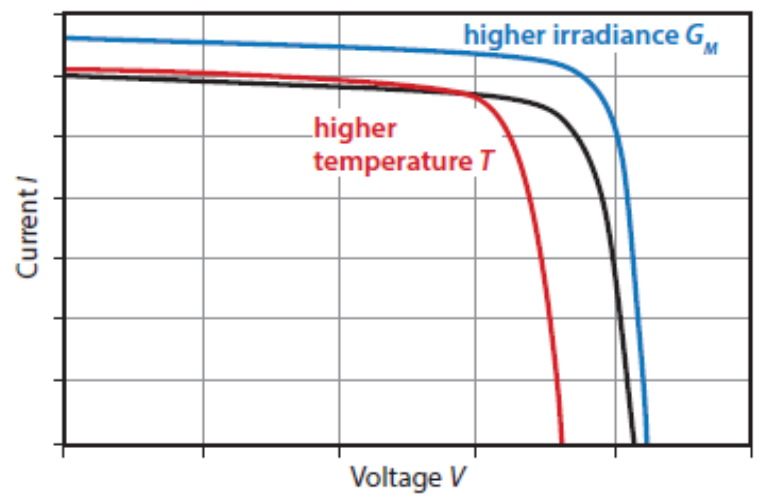

Figura 1 Curva de corriente y voltaje Fuente: Edx Solar energy

En la Figura 2 se puede observar el comportamiento del voltaje-corriente con respecto a la temperatura de operación de una celda solar, la disminución de la potencia con respecto al aumento de la temperatura y la disminución del voltaje de circuito abierto de la misma celda con el aumento de la temperatura, las cuales pueden resumirse en las razones de cambio (1).

$\frac{d P}{d T}, \frac{d V_{o c}}{d T}$

$P$ es la potencia en Watts, $V o c$ es el voltaje en circuito abierto.
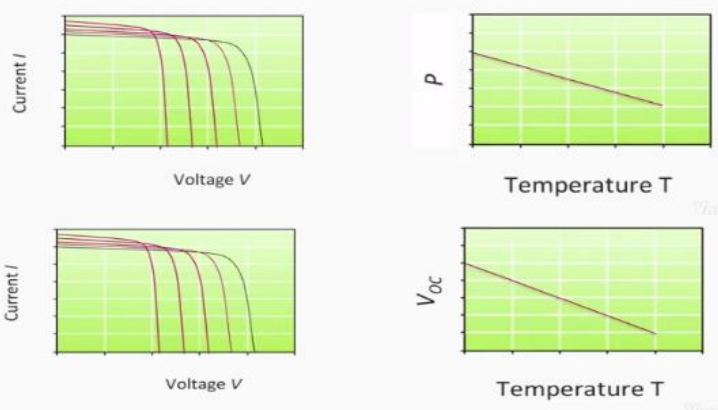

Figura 2 Curva de I-V incrementando el valor de la temperatura e irradiación constante Fuente: Edx Solar energy 
Con el coeficiente de temperatura, se puede calcular la potencia de salida del panel solar con la relación:

$$
\begin{aligned}
& P(T)=P_{S T C}+\frac{d P}{d T}\left(T-T_{S T C}\right) \\
& V O C(T)=V o c+\frac{d V o c}{d T}\left(T-T_{S T C}\right)
\end{aligned}
$$

La relación $\frac{d P}{d T}$ denota el coeficiente de temperatura y la temperatura de referencia es de $25^{\circ} \mathrm{C}$. (Romero, 2002) ha mostrado que la curva I-V de una celda solar a distintas temperaturas e igual irradiación, presenta una disminución del voltaje al incrementar la temperatura, que ha modelado con la relación (2). Por otra parte (E. E. Granda-Gutiérrez, 2013), ha desarrollado la simulación que, al incrementar la temperatura de la celda, el punto máximo de potencia disminuye, debido a una importante reducción del voltaje de salida, que se debe al incremento en la agitación térmica de los portadores de carga en el semiconductor, lo que se manifiesta como reducción en el potencial de unión.

(Alonso García \& Balenzategui, 2004), mencionan que la diferencia de temperatura del módulo y la temperatura ambiente (Tj-Tamb), con respecto a la irradiación $\left(\mathrm{W} / \mathrm{m}^{2}\right)$, es que, a mayor irradiación, se tiene mayor diferencia de temperatura (Tj-Tamb), cuando la temperatura ambiente es de $20^{\circ} \mathrm{C}$. Para calcular la temperatura del módulo, propusieron la siguiente relación:

$T_{m}=T_{a m b}+(N O C T-20) \frac{E}{800}$

Donde: $T m$ es el Módulo temperatura, Tamb es la temperatura ambiente, NOCT: Operación Normal de temperatura de la celda y $E$ es la irradiación en $\mathrm{W} / \mathrm{m}^{2}$.

\section{Características de la celda solar}

Las celdas solares que se utilizaron para el análisis de su eficiencia son celdas solares policristalinas de $52.8 \mathrm{~mm} \times 52.8 \mathrm{~mm} \times 0.22$ $\mathrm{mm}$, de $0.5 \mathrm{~V}, 0.1$ A y $0.05 \mathrm{~W}$, sus condiciones de operación normal de la celda son: NOCT $20^{\circ} \mathrm{C}$ (Operación Normal de temperatura de la celda) a $1.5 \mathrm{AM}$ (masa de aire) con una irradiación $\mathrm{G}=1 \mathrm{~kW} / \mathrm{m}^{2}$ y la velocidad del viento no mayor a $1 \mathrm{~m} / \mathrm{s}$. Se adquirieron a la empresa jlsolars.com del país de China.

\section{Características del disipador de solar}

Las características del disipador de calor son: una resistencia térmica de $2.4^{\circ} \mathrm{C} / \mathrm{W}$, y sus dimensiones de altura: 50 x 50 x $25 \mathrm{~mm}$, fabricado en aluminio, fabricante: Fischer elktronik.
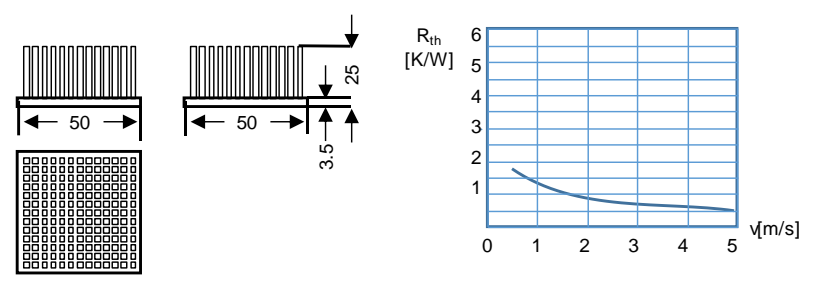

Figura 3 Disipador de calor

Fuente: https://mexico.newark.com/fischer-elektronik

\section{Metodología}

\section{Identificación de la ubicación y posición de la celda solar:}

Un factor importante en la eficiencia de las celdas solares, es la temperatura de operación, se pretende identificar si al incrementar la temperatura de celda solar, hay un cambio en su eficiencia.

Para conocer su producción de potencia eléctrica, se aplicó una carga, y se registró en un equipo de adquisición de datos, se hizo uso de métodos cuantitativos, para conocer el comportamiento de sus los parámetros eléctricos. Para los experimentos se trabajó con dos tipos de celdas, una celda solar simple y una celda solar con un disipador de calor.

Se diseñó e implementó una estructura para colocar un disipador de calor en la cara posterior de una de las celdas solares. Se identificó la ubicación y posición de la celda solar, para lo que el ángulo de inclinación debe ser igual a la latitud (distancia angular entre la línea ecuatorial y un punto determinado de la Tierra), lo que asegura la máxima cantidad de luz solar expuesta durante el día, que para la ciudad de Cancún, sus Coordenadas geográficas son: Latitud: $21^{\circ} 10^{\prime} 27^{\prime \prime} \quad \mathrm{N}$. Longitud: $86^{\circ} 50^{\prime} 47^{\prime \prime} \mathrm{O}$, por lo tanto las celdas solares se colocaron a $21^{\circ}$, siendo la mejor orientación de los paneles solares hacia el sur, debido a que en el equinoccio se tiene el día con más sol, de 6:00 a 18:00 horas. 


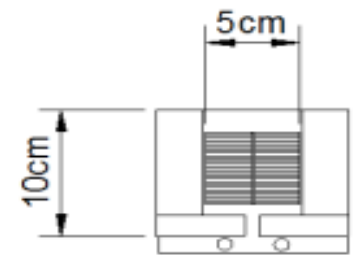

Vista superior de la celda solar
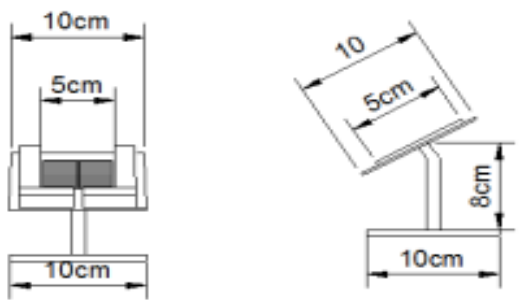

Figura 4 Celda solar de $52.8 \mathrm{~mm}$ x $52.8 \mathrm{~mm}$ x $0.22 \mathrm{~mm}$ de $0.5 \mathrm{~V}, 0.1$ A y $0.050 \mathrm{~W}$ y diseño de la base, con disipador de calor.

Fuente: Elaboración propia del diseño

El diseño de la base para las celdas solares que se aprecia en la Figura, tiene el propósito de mantenerlas fijas a la orientación antes mencionada, se construyeron con material de trovicel, cuyas características técnicas son: la resistencia a la fricción, elasticidad y con resistencia a altas temperaturas ambientales.

\section{Registro de parámetros eléctricos}

La celda solar es sometida a la irradiación solar en un horario de 9:00 am a las 17:00 hrs durante el mes de julio, y sus parámetros eléctricos (corriente, voltaje y la temperatura), son registrados través de los siguientes equipos de adquisición de datos:

1. Fuente de carga eléctrica de CD de 300 watts BK PRECISION Serie 8500, el cual aplica una carga electrónica a la celda solar, es decir demanda una carga específica de operación, para conocer su corriente, voltaje y potencia.

2. Equipo de adquisición de datos de 20 canales Keysight 34970A, para registrar las mediciones de voltaje generado por la celda solar, la temperatura de la celda solar y el voltaje generado por el piranómetro, encargado de registrar la irradiación solar que incide en la celda solar.

3. Equipo de Radiación solar piranómetro marca Eppley modelo 8-48 con factor de conversión de: $775 \times 10-6 \mathrm{~W} / \mathrm{m}^{2}$, el cual se empleó para la medición de la irradiación, incidente sobre la superficie del área de estudio.
4. Equipo anemómetro de hilo caliente a temperatura constante para mediciones de aire en túnel de viento para registrar la velocidad del mismo.

\section{Resultados}

\section{Operación de la celda solar}

Una de las celdas de $0.5 \mathrm{~V}, 0.1$ A y $0.05 \mathrm{~W}$, se conectó al equipo de Carga eléctrica de CD de 300 watts BK PRECISION Serie 8500, y se programó para que demande un flujo de corriente eléctrica de $0.1 \mathrm{~A}$, esta carga eléctrica, la estará soportando en función de la irradiación solar.

\section{Análisis de datos}

La temperatura de la celda solar, considerando la velocidad del viento se pude expresar como:

$T M=T a+\frac{T_{N O C T}-20^{\circ}}{800} G M\left(\frac{9.5}{5.7+3.8 \cdot w}\right)\left(1-\frac{n_{c e l l}}{T \propto}\right)$

Donde: TM es la temperatura nominal de la celda en ${ }^{\circ} \mathrm{C}$, w es la velocidad del viento, $\mathrm{T}$ es la transmitancia de la parte frontal del módulo, $\alpha$ medida de absorción de radiación del módulo, T $\alpha$ es la fracción de incidencia de luz, que es absorbida por la celda solar, usualmente es de 0.9 .

A continuación, se presentan en la Tabla 1 las medidas estadísticas registradas en el experimento.

\begin{tabular}{|c|c|c|c|c|c|}
\hline Medición & $\begin{array}{l}\text { Tem. } \\
\text { celda }\end{array}$ & $\begin{array}{l}\text { Tem. } \\
\text { ambiente }\end{array}$ & $\begin{array}{l}\text { Diferencia } \\
\text { de Tem. }\end{array}$ & Irrad. & $\begin{array}{l}\text { Viento } \\
(\mathrm{m} / \mathrm{s})\end{array}$ \\
\hline Estadística & $\begin{array}{l}(\mathrm{Tc}, \\
\left.{ }^{\circ} \mathrm{C}\right)\end{array}$ & $\left(\mathrm{Ta}{ }^{\circ} \mathrm{C}\right)$ & $\mathrm{Tc}-\mathrm{Ta}\left({ }^{\circ} \mathrm{C}\right)$ & $\left(\mathrm{W} / \mathrm{m}^{2}\right)$ & \\
\hline Media & 40.4 & 31.77 & 8.65 & 675.47 & 1.8 \\
\hline Mediana & 42.03 & 30.79 & 8.33 & 670.79 & 1.7 \\
\hline Moda & $45^{\mathrm{a}}$ & $30^{\mathrm{a}}$ & $10^{\mathrm{a}}$ & $62^{\mathrm{a}}$ & $0^{\mathrm{a}}$ \\
\hline $\begin{array}{l}\text { Desviación } \\
\text { estándar }\end{array}$ & 8.1 & 2.9 & 8.1 & 365.4 & 1.13 \\
\hline Varianza & 67.2 & 8.8 & 65.9 & 133566 & 1.2 \\
\hline Mínimo & 22 & 27 & -10 & 54 & 0 \\
\hline Máximo & 54 & 40 & 25 & 1289 & 6 \\
\hline
\end{tabular}

Tabla 1 Estadístico descriptivo de la temperatura, viento e irradiación solar de 2391 muestras, que van de 09 hrs a $17 \mathrm{hrs}$

Fuente: Elaboración Propia

A partir de los datos registrados de temperatura de la celda solar, temperatura ambiente, irradiación solar y viento, se hizo un estadístico descriptive. 
Cuyos resultados se muestran en la tabla 1 , y se muestra que la diferencia de temperatura entre la temperatura de la celda solar y la temperatura ambiente es de $-10^{\circ} \mathrm{C}$ por debajo y $25^{\circ} \mathrm{C}$ por encima de la temperatura ambiental.

En la Figura, se muestra la temperatura de la celda solar, que inicia a una temperatura de $22^{\circ} \mathrm{C}$ y va incrementado su valor al paso del tiempo, alcanzando una temperatura máxima de $54^{\circ} \mathrm{C}$ alrededor de las 3:00 pm.

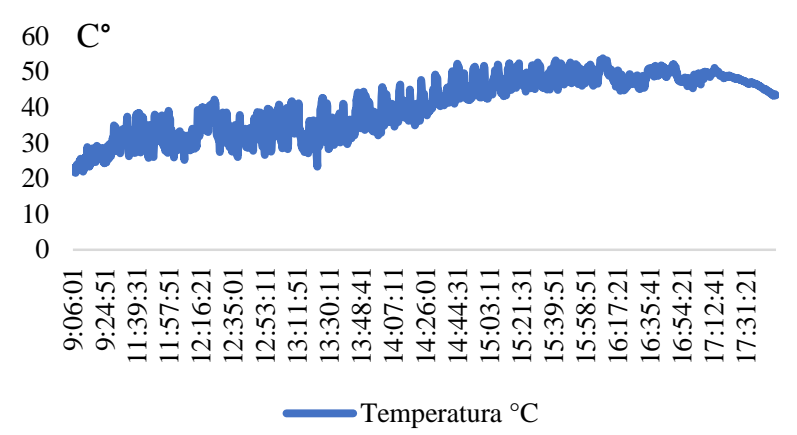

Figura 5 Temperatura de la celda solar Fuente: Elaboración Propia

Del mismo modo la diferencia de temperaturas de la celda solar y la temperatura ambiente, (Tc-Ta) mantiene un incremento lineal, con variaciones debidas a factores como la irradiación solar y el viento, tal como se puede apreciar en la Figura 6. También se puede observar que en la variación de la diferencia de la temperatura Tc-Ta con respecto al viento, al incrementar este último, disminuye la diferencia Tc-Ta, otro dato que se puede apreciar es que la velocidad promedio del viento fue de $1.88 \mathrm{~m} / \mathrm{s}$, velocidad máxima de $6 \mathrm{~m} / \mathrm{s}$ y velocidad mínima de $0 \mathrm{~m} / \mathrm{s}$.

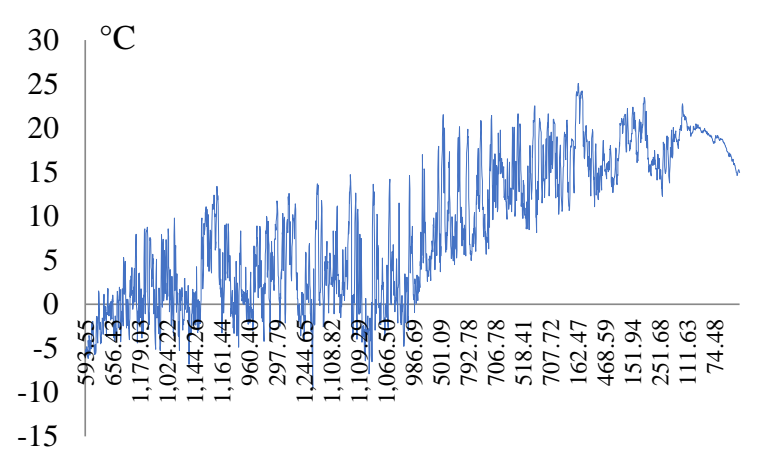

Figura 6 Gráfica de la diferencia de temperatura de la celda solar y la temperatura ambiente Tc-Ta Fuente: Elaboración Propia

En la celda solar con el disipador de calor, se registraron las mediciones eléctricas de voltaje, corriente, potencia, temperatura $\mathrm{e}$ irradiación solar.
La potencia de la celda solar disminuye al incrementar el valor de la diferencia de temperatura Tc-Ta. Monitoreando la velocidad del viento y la diferencia de temperatura $\mathrm{Tc}-\mathrm{Ta}$, al incrementar la velocidad del viento, disminuye la diferencia de temperatura Tc-Ta e incrementa el valor de la potencia, que se puede observar en la Figura 7.

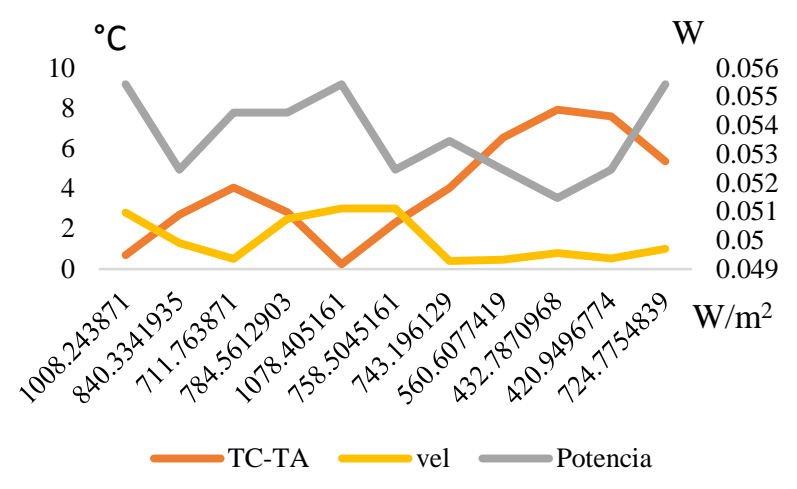

Figura 7 Comportamiento de la potencia de la celda solar, respecto de la diferencia de temperatura de la celda, la temperatura ambiente y la velocidad del viento.

Fuente: Elaboración Propia

Considerando la corriente de corto circuito Isc y el voltaje en circuito abierto Voc, se puede generar la curva I-V (como se observa en la Figura 8) conociendo la irradiación solar y la temperatura, con la relación propuesta por (Romero, 2002):

$I s c_{2}=I s c_{1}\left(\frac{H_{2}}{H_{1}}\right)+\alpha\left(T_{2}-T_{1}\right) T s c_{1}$
$V o c_{2}=V o c_{1}+N \times \beta\left(T_{2}-T_{1}\right)+N \times V t_{1} \ln \frac{H_{2}}{H_{1}}$

(7)

$V_{T 2}=V t_{1} \frac{T_{2}}{T_{1}}$

La celda solar tiene las siguientes características: Isc $=0.1 \mathrm{~A}, \mathrm{Voc}=0.5 \mathrm{~V}, \mathrm{~N}=1$, Viento promedio de $1.88 \mathrm{~m} / \mathrm{s}$, y Temperatura ambiente promedio de: $31.77^{\circ} \mathrm{C}$, con un rango de irradiación de $54 \mathrm{~W} / \mathrm{m}^{2}$ a $1289 \mathrm{~W} / \mathrm{m}^{2}$.

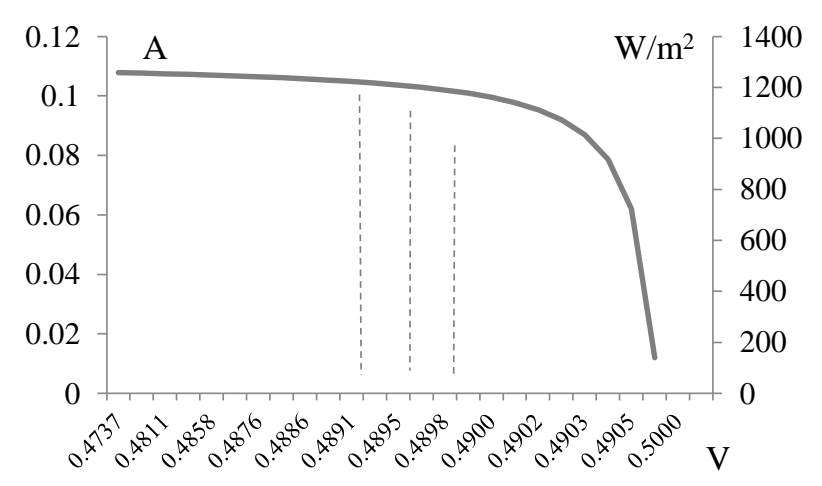

Figura 8 Curva I-V, a distinta irradiación y temperatura Fuente: Elaboración Propia 
El comportamiento de la diferencia de temperatura Tc-Ta como se aprecia en la Figura 9, se puede conocer en función de la intensidad de radiación solar y de la velocidad del viento, y pueden aproximarse según las ecuaciones desarrolladas por (Romero, 2002):

$$
\begin{aligned}
& T c-T a=\frac{q}{h} \\
& h=a_{o}+b_{o} v
\end{aligned}
$$

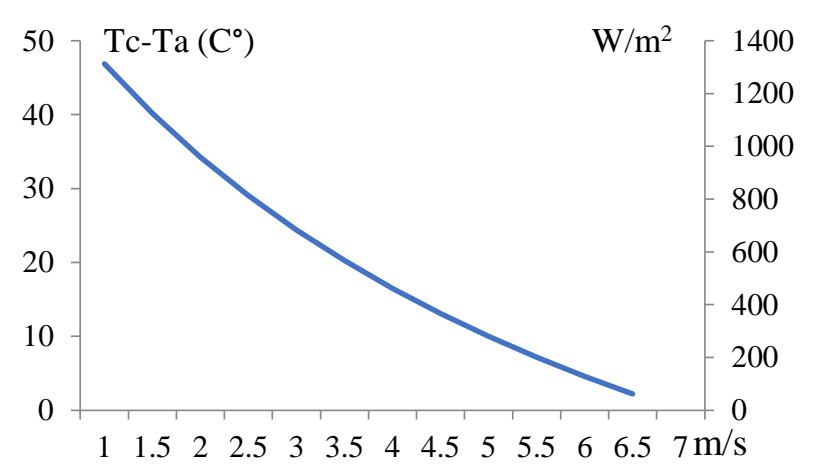

Figura 9 Relación del Tc-Ta, con respecto a la velocidad del viento y la irradiación solar teórica.

Fuente: Elaboración Propia

Con valores de los coeficientes: $a_{o}=$ $22, b_{o}=3.6$ y Tc-Ta siendo la diferencia de temperatura de la celda solar y la temperatura ambiente, q la irradiación solar, $v$ la velocidad del viento y $h$ el coeficiente de transmisión térmica. Aplicando la relación (9), se observa que la velocidad del viento tiene un efecto importante en la disminución de la temperatura de la celda solar. En condiciones reales, la irradiación solar, la temperatura y la velocidad del viento, es variable, sin embargo, con las mediciones realizadas, se puede observar en la Figura 10, como al incrementar la velocidad del viento, disminuye la diferencia de la temperatura Tc-Ta, también en función del valor de la irradiación solar. Por otra parte, se observa que, a mayor irradiación, se tiene mayor diferencia de temperatura (Tc-Ta).

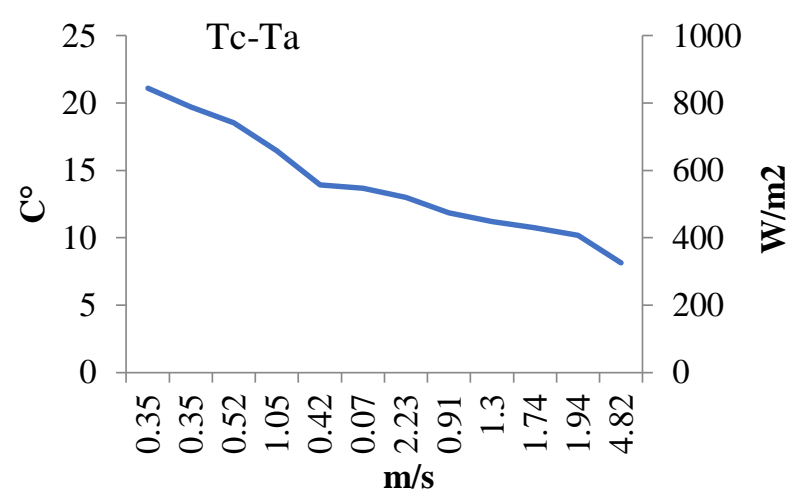

Figura 10 Relación del Tc-Ta, con respecto a la velocidad del viento y la irradiación solar en condiciones reales Fuente: Elaboración Propia
Utilizando la relación (2) para calcular la potencia en la celda solar, con un coeficiente de temperatura:

$\frac{d P}{d T}=-0.0004 /{ }^{\circ} \mathrm{C}$ se tiene:

$P(T)=P_{S T C}+\frac{d P}{d T} \times\left(T-T_{S T C}\right)$

$X=V_{O C}, P, V_{S C}$,

$T=54^{\circ} \mathrm{C}$, es la temperatura de la celda

$T_{S T C}=25^{\circ} \mathrm{C}$, es la temperatura de operación

$P(T)=0.05 W_{S T C}+\left(-\frac{0.0004 \mathrm{~W}}{{ }^{\circ} \mathrm{C}}\right) \times\left(54^{\circ} \mathrm{C}-25^{\circ} \mathrm{C}_{S T C}\right)$

$P(T)=0.038 W$

Como se puede observar el signo del coeficiente de temperatura, determina si incrementa o disminuye la potencia de salida de la celda solar. En la Figura 3, se muestra la potencia de salida y la temperatura de la celda solar, y de acuerdo al estadístico descriptivo se tiene que; la temperatura de la celda solar tiene una media de $42.03^{\circ} \mathrm{C}$, una mínima de $22^{\circ} \mathrm{C}$ y máxima de $54^{\circ} \mathrm{C}$, y una potencia media de: $0.0486 \mathrm{~W}$ una mínima de $0.02 \mathrm{~W}$ y máxima de $0.06 \mathrm{~W}$. Sin embargo en la celda solar, se mantiene la potencia en un promedio de 0.0486 W hasta llegar a una temperatura de $53.7^{\circ} \mathrm{C}$ y que puede apreciarse en la Figura 4, donde la potencia disminuye a $0.035 \mathrm{~W}$ que se acerca al cálculo de la potencia con la relación $(2), P(T)=$ $0.038 \mathrm{~W}$

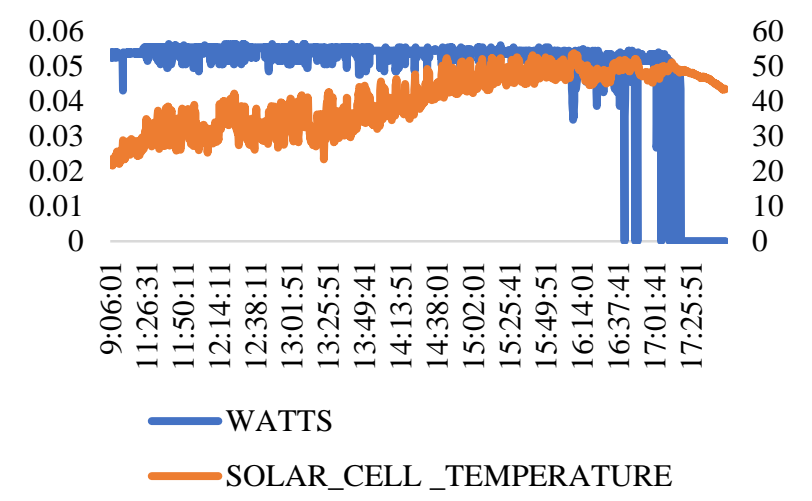

Figura 3 Temperatura y potencia de salida de la celda solar

Fuente: Elaboración Propia

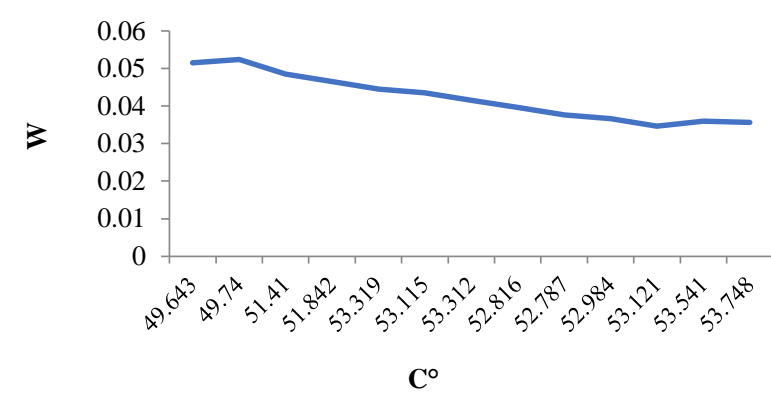

Figura 4 Relación de la potencia de salida de la celda solar y la temperatura

Fuente: Elaboración propia 
De acuerdo a las mediciones realizadas, se identifica que el comportamiento de la potencia de salida de la celda solar, tiene la tendencia de la relación (2), en función del coeficiente de temperatura $\frac{d P}{d T}$ y la temperatura de operación de la celda solar $\mathrm{T}$, que a $53.74^{\circ} \mathrm{C}$, disminuye su potencia a un valor de $0.03564 \mathrm{~W}$

Al incrementar el valor de la temperatura, disminuye la potencia de salida de la celda solar. En función del coeficiente de temperatura y la diferencia de $T-T_{S T C}$.

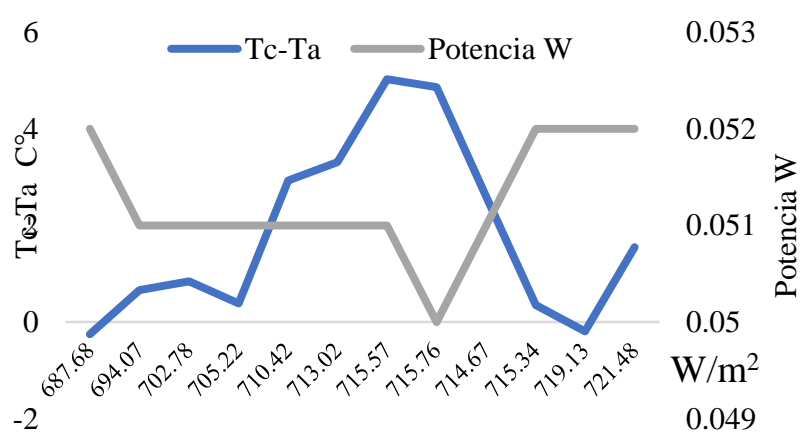

Figura 13 Comportamiento de la potencia de la celda solar sin disipador, respecto de la diferencia de temperatura de la celda solar y la temperatura ambiente

Fuente: Elaboración Propia

En la figura 13 se aprecia que la potencia incrementa cuando disminuye la diferencia de temperatura Tc-Ta, aun cuando hay un incremento de la irradiación solar.

\section{Comparación de la eficiencia de la celda solar con disipador y sin disipador}

\begin{tabular}{|c|c|c|c|c|c|}
\hline Potencia (W) & $\mathbf{N}$ & Mínimo & Máximo & Media & $\begin{array}{l}\text { Desviación } \\
\text { estándar }\end{array}$ \\
\hline on Disipador & 2218 & .00 & .06 & .0524 & .00663 \\
\hline$\overline{n d}$ & 2218 & .00 & .05 & .0506 & .00868 \\
\hline & 2218 & & & & \\
\hline
\end{tabular}

Tabla 2 Estadístico descriptivo de la potencia de la celda con disipador y sin disipador

Fuente: Elaboración Propia

En la tabla 3, se muestra la prueba Anova, para conocer si hay diferencia en la producción de potencia eléctrica entre, la celda solar con disipador y la celda solar sin disipador. El resultado muestra que, el nivel de significancia (0.000) es menor a 0.05 , entonces, se rechaza que sean de medias iguales y se acepta que son diferentes, es decir, la eficiencia de las celdas solares es diferente usando el disipador de calor. Al disminuir la temperatura de la celda solar, se encontró que mejora su eficiencia del 3.4 al $5.4 \%$

\begin{tabular}{|c|c|c|c|c|c|c|}
\hline & \multicolumn{3}{|c|}{ ANOVA } & \multirow[b]{2}{*}{$\mathrm{F}$} & \multirow[b]{2}{*}{ Sig } \\
\hline & & $\begin{array}{l}\text { Suma de } \\
\text { cuadrados }\end{array}$ & $\mathrm{gl}$ & $\begin{array}{c}\text { Media } \\
\text { cuadrática }\end{array}$ & & \\
\hline \multicolumn{2}{|c|}{ Inter sujetos } & .125 & 2217 & .000 & & \\
\hline \multirow[t]{3}{*}{$\begin{array}{l}\text { Intra } \\
\text { sujetos }\end{array}$} & \begin{tabular}{|l|}
$\begin{array}{l}\text { Entre } \\
\text { elementos }\end{array}$ \\
\end{tabular} & .004 & 1 & .004 & 61.59 & .000 \\
\hline & Residuo & .139 & 2217 & .000 & & \\
\hline & Total & .143 & 2218 & .000 & & \\
\hline \multicolumn{2}{|l|}{ Total } & .268 & 4435 & .000 & & \\
\hline
\end{tabular}

Tabla 5 Prueba Anova de potencia eléctrica con disipador y sin disipador de calor en la celda solar

Fuente: Elaboración Propia

\section{Conclusiones}

En la ciudad de Cancún, las celdas solares empleadas con disipador de calor, sometidas a operación en las condiciones ambientales; temperatura ambiente en promedio de $31.77^{\circ} \mathrm{C}$, irradiación solar promedio de $675.47 \mathrm{~W} / \mathrm{m}^{2} \mathrm{y}$ con una velocidad del viento de $1.88 \mathrm{~m} / \mathrm{s}$, registra un voltaje promedio de $0.4911 \mathrm{~V}$, un voltaje mínimo y máximo de $0.00 \mathrm{~V}, \quad 0.57 \mathrm{~V}$ respectivamente, una potencia promedio de $0.0524 \mathrm{~W}$, la temperatura media, mínima $\mathrm{y}$ máxima es de $40.42^{\circ} \mathrm{C}, \quad 22^{\circ} \mathrm{C}$ y $54^{\circ} \mathrm{C}$ respectivamente.

Como se puede ver en la figura No. 10; al incrementar la velocidad del viento, disminuye la diferencia de temperatura Tc-Ta e incrementa el valor de la potencia. También se muestra en la gráfica No. 9 que, al incrementar la temperatura de la celda solar, disminuye la potencia, debido al coeficiente de temperatura y la temperatura máxima de operación. Sin embargo, su producción de energía se mantiene en un promedio de: $0.0524 \mathrm{~W}$, cuando la celda solar alcanza la temperatura máxima registrada de $53.74^{\circ} \mathrm{C}$, disminuye su eficiencia al $71 \%$.

Con respecto a la comparación de la potencia de la celda solar con disipador y sin disipador, a través de la prueba Anova, se obtiene un nivel si significancia menor de 0.05 , con lo que se rechaza que sean potencias de medias iguales y se acepta que son diferentes y mejora su eficiencia en un rango del 3.4 al $5.4 \%$

\section{Agradecimientos}

Para llevar a cabo está investigación, se requirieron recursos económicos para la adquisición de equipo de laboratorio, que fue adquirido por la aprobación de la red temática de colaboración académica de la convocatoria de PRODEP. 
Un agradecimiento especial al Dr. Frédéric J. Lesage del Département d'informatique et d'ingénierie, Université du Québec en Outaouais por su dirección y recomendaciones para la realización de este proyecto.

\section{Referencias}

Alonso García, M. C., \& Balenzategui, J. L. (2004). Estimation of photovoltaic module yearly temperature and performance based on Nominal Operation Cell Temperature calculations. Renewable energy, 29(12), 19972010.

Arno Smets, K. J. (2016). Solar Energy, t. p. Cambridge: UIT Cambridge.

E. E. Granda-Gutiérrez, O. A.-G. (2013). MODELADO Y SIMULACIÓN DE CELDAS $\mathrm{Y}$ PANELES SOLARES. Congr. Int. Ing. Electrón. Mem. Electro, 35, 17-22.

Lesage, F. S. (Marzo de 2017). Eficiencia de las celdas solares. Revista de Aplicaciones de la Ingenieria, 4(19), 19-27. Obtenido de www.ecorfan.org/bolivia

Organización meteorológica mundial. (7 de Mayo de 2019). Servicio de información meteorológica mundial, predicciones oficiales. Obtenido de http://wwis.aemet.es/es/city.html?cityId=1209

Romero, F. C. (2002). Cálculo de la Temperatura de Operación de Células Solares en un Panel Fotovoltaico Plano. Departamento de Energías Renovables. MADRID, ESPAÑA: Editorial CIEMAT.

S. Mekhilef, R. S. ( 2012/6.). Effect of dust, humidity and air velocity on efficiency of photovoltaic cells. Renewable Sustainable Energy, 16(5), 2920-2925. 